\title{
Patrones de distribución geográfica de los mamíferos de Jalisco, México
}

\author{
Mammal geographic distribution patterns in Jalisco State, Mexico
}

\author{
Ismael Ramos-Vizcaíno, Sergio Guerrero-Vázquez y Francisco Martín Huerta-Martínez* \\ Centro Universitario de Ciencias Biológicas y Agropecuarias (CUCBA), Universidad de Guadalajara. Km 15.5 carretera a Nogales, Las Agujas \\ Nestipac, Zapopan, Jalisco. México \\ *Correspondencia: fhuerta@cucba.udg.mx
}

\begin{abstract}
Resumen. Se analizaron los patrones de distribución de la riqueza de mamíferos en el estado de Jalisco y sus relaciones con algunos factores ambientales. Se obtuvo información de registros de mamíferos de Jalisco de diferentes colecciones biológicas nacionales y extranjeras. Se utilizaron las localidades de recolecta para realizar una cobertura de puntos. El estado se dividió en 159 unidades de clasificación geográfica (UCG's) de 15' por 15'. Se sobrepuso la cobertura de puntos, las UCG y algunos mapas de CONABIO para formar una matriz de presencia-ausencia. Se analizó la distribución de la riqueza por tipo de vegetación y altitud. Se observó un gradiente de riqueza que va desde las zonas tropicales hasta las semiáridas. Por altitud, la mayor riqueza se encontró entre los 1500 y 2000 m y la menor de los 4000 a $4500 \mathrm{~m}$. Se aplicó una ordenación de Bray-Curtis y una clasificación conTWINSPAN. Ambas fueron consistentes en formar 2 grupos de mamíferos; uno con especies de la costa y el otro del noreste del estado, lo que refleja un gradiente climático. El porcentaje de variación acumulada fue del 94\% y las variables del medio con mayor influencia fueron precipitación, temperatura, evaporación, altitud y vegetación.
\end{abstract}

Palabras clave: Bray-Curtis, riqueza, clasificación, biogeografía

\begin{abstract}
We analyzed the patterns of distribution of mammal species richness in Jalisco State and their relationships with some environmental factors. We retrieved distribution data from several national and foreign biological collections. We used the collecting localities to generate a spatial record of distribution points. The state was divided into 159 geographic units of classification (GUC's) of 15' by $15^{\prime}$. We overlap the point cover, GUCs and some maps from CONABIO to create a presence-absence matrix. We analyzed the richness distribution by vegetation type and elevation. A richness gradient was observed from tropical to semiarid vegetation types and according to elevation; we observed higher richness between 1500 and $2000 \mathrm{~m}$ a. s. 1 . and lower richness between 4000 to 4500 . A Bray-Curtis ordination and a classification TWINSPAN were used. They were consistent in separating two groups of species: one of them including coastal species and the other with northeastern species, reflecting a climatic gradient. The percentage of accumulated variance was $94 \%$ and precipitation, temperature, evaporation, elevation and vegetation were the most important environmental variables.
\end{abstract}

Key words: Bray-Curtis, richness, classification, biogeography

\section{Introducción}

México es el país más diverso en América en cuanto a mamíferos se refiere y ocupa el segundo lugar en el mundo después de Indonesia (Ceballos et al., 2002a). Esta diversidad ha sido explicada como resultado de su historia geológica, clima, topografía y tipos de vegetación (Álvarez y Lachica, 1974; Ceballos y Navarro, 1991; Fa y Morales, 1993). De acuerdo con Ramírez-Pulido et al. (2005), existen 475 especies de mamíferos terrestres. Si

Recibido: 24 febrero 2006; aceptado: 22 noviembre 2006 agregamos los mamíferos marinos, México alberga 522 especies (Ceballos et al., 2002b). Aunque la diversidad de mamíferos se compone principalmente de roedores y murciélagos, en el país también están representados los órdenes Didelphimorphia, Cingulata, Lagomorpha, Carnivora, Primates, Soricomorpha y Artiodactyla, entre otros (Fa y Morales, 1993; Ceballos et al., 2002a).

El estado de Jalisco se localiza en una zona de transición biológica entre las regiones neártica y neotropical (Palomera-García et al., 1994; Ortega y Arita, 1998), con la presencia de especies que se distribuyen en ambas, por lo cual está considerado zona de confluencia de especies 
(Guerrero et al., 1995; Guerrero y Cervantes, 2003). A la accidentada topografía de Jalisco, dada la confluencia de la Sierra Madre Occidental, la Sierra Madre del Sur, el Eje Neovolcánico, la depresión del Balsas y la mesa central se debe la gran heterogeneidad ambiental y climática lo que permite, a su vez, la presencia de diferentes factores, como aridez (Fa y Morales, 1993), barreras geográficas, latitud (Fa y Morales, 1993; Kaufman, 1995; Badgley y Fox, 2000; Hawkins et al., 2003; Iñiguez y Santana, 2005), productividad (Rosenzweig, 1992; Hawkins et al., 2003), clima (Porter et al., 2000; Hawkins et al., 2003) y elevación (Fa y Morales, 1993; Badgley y Fox, 2000; Lomolino, 2001), que influyen en los patrones de distribución de los mamíferos a grandes escalas, mientras que los factores locales de competencia, depredación, mutualismo, disponibilidad de alimento (Rose y Polis, 1998), temperatura (Graham, 1983; Virgós et al., 2001), heterogeneidad del hábitat, y la cobertura, densidad y estructura de la vegetación se registran como responsables de los patrones de riqueza (Ceballos y Miranda, 1986; Kelt et al., 1999; Bakker y Kelt, 2000; Williams et al., 2002). Así, la heterogeneidad de hábitat que produce el relieve y la presencia de especies de origen neártico y neotropical dan como resultado que el estado tenga una alta diversidad de mamíferos (Guerrero et al, 1995; Escalante et al., 2002; Guerrero y Cervantes, 2003; Iñíguez y Santana, 2005). Además de la diversidad, existen en Jalisco regiones con un alto endemismo en mamíferos; tales son los casos de la Sierra Madre Occidental y el Eje Neovolcánico (Ceballos y Rodríguez, 1993; Fa y Morales, 1993; Guerrero et al., 1995; Ceballos et al., 1998).

En todo el mundo los mamíferos muestran un gradiente latitudinal en diversidad de especies (Kaufman, 1995, Kaufman y Willig, 1998), el cual se explica por variables ambientales (Currie, 1991; Kerr y Packer, 1997; Badgley y Fox, 2000; Hawkins et al., 2003). Sin embargo, existen diferencias en los factores que influyen en la distribución de un determinado taxón, dependiendo de la escala del estudio. En una escala mayor, las variables que mejor explican la distribución de un taxón pueden diferir de aquellas que explicarían la distribución del mismo taxón a una escala menor (Collins y Glenn, 1997; Badgle y Fox, 2000; Morrone y Ruggiero, 2000; Mittelbach et al., 2001; Whittaker et al., 2001; Willis y Whittaker, 2002; Morrone y Escalante, 2002; Hawkins et al., 2003; Willig et al., 2003; Steiner y Leibold, 2004). También existen diferencias en los factores que influyen en la distribución, dependiendo de los taxa analizados y de la latitud en la cual se distribuyen (Badgley y Fox, 2000; Hawkins et al., 2003).

En el presente trabajo se proporciona información acerca del patrón general de distribución de las especies de la clase Mammalia en Jalisco y se muestra la relación de dicho patrón con algunos factores ambientales. La finalidad de esta información es contribuir con la realización de propuestas y estrategias de conservación y manejo de los mamíferos.

\section{Materiales y métodos}

Zona de estudio. Jalisco se encuentra entre los $18^{\circ} 15^{\prime} 05^{\prime}$, y $20^{\circ} 51^{\prime} 49^{\prime \prime}$ ' $\mathrm{N}$ y los 101'28' $15^{\prime \prime}$ ' y $105^{\circ} 43^{\prime} 18^{\prime \prime}$ ' O, en el occidente de México. El estado tiene una superficie de 80 $137 \mathrm{~km}^{2}$ que representan el $4 \%$ de la superficie del país (Fig. 1). Limita al norte con los estados de Zacatecas, Aguascalientes, Durango y San Luis Potosí, al este con Michoacán y Guanajuato, al noroeste con Nayarit, al sur con Colima y al oeste con el océano Pacífico. De acuerdo con Rzedowski (1986) tiene 13 tipos diferentes de vegetación, de los cuales el bosque tropical caducifolio y el bosque de pino encino son los que cubren una mayor superficie. En Jalisco confluyen el Eje Neovolcánico Transversal, la Sierra Madre Occidental, la Sierra Madre del Sur, la depresión del Balsas y la Mesa Central; esto provoca una variación altitudinal que va desde el nivel del mar hasta los $4500 \mathrm{~m}$. En Jalisco se presentan 2 estaciones claramente marcadas: la temporada de lluvia, que se extiende de junio a septiembre y la temporada de estiaje, que abarca los meses restantes. Los valores de precipitación oscilan entre los $400 \mathrm{~mm}$ por año en la región noreste del estado y $1700 \mathrm{~mm}$ en las regiones montañosas cercanas a la costa (INEGI, 1981). Presenta un gradiente térmico que disminuye de sur a norte y siempre en relación con la altitud (Zamudio et al., 1987). En general, los climas cálidos se localizan a lo largo de la franja costera; en la zona del centro son más frecuentes los semicálidos; en las partes altas de los sistemas montañosos dominan los climas templados, y los semisecos en la parte norte y noroeste; por último, el clima semifrío se presenta en el área del Nevado de Colima (INEGI, 1981) (Figura 1).

Patrones de distribución de la riqueza. Con el propósito de tener la representación de unidades de muestreo de la misma superficie, el estado se dividió en 159 cuadros o unidades de clasificación geográfica (UCG) de 15 minutos por 15 minutos, que representan aproximadamente

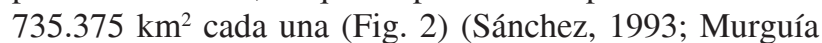
y Rojas, 2001).

Se obtuvieron datos de registros de los mamíferos depositados en diferentes colecciones zoológicas nacionales y extranjeras que estuvieran distribuidos dentro de las UCG en las que se dividió la superficie del estado. Para las UCG que sólo abarcaron una porción del estado, los registros se buscaron en los estados que colindan con Jalisco. Con los registros de mamíferos se elaboró una 


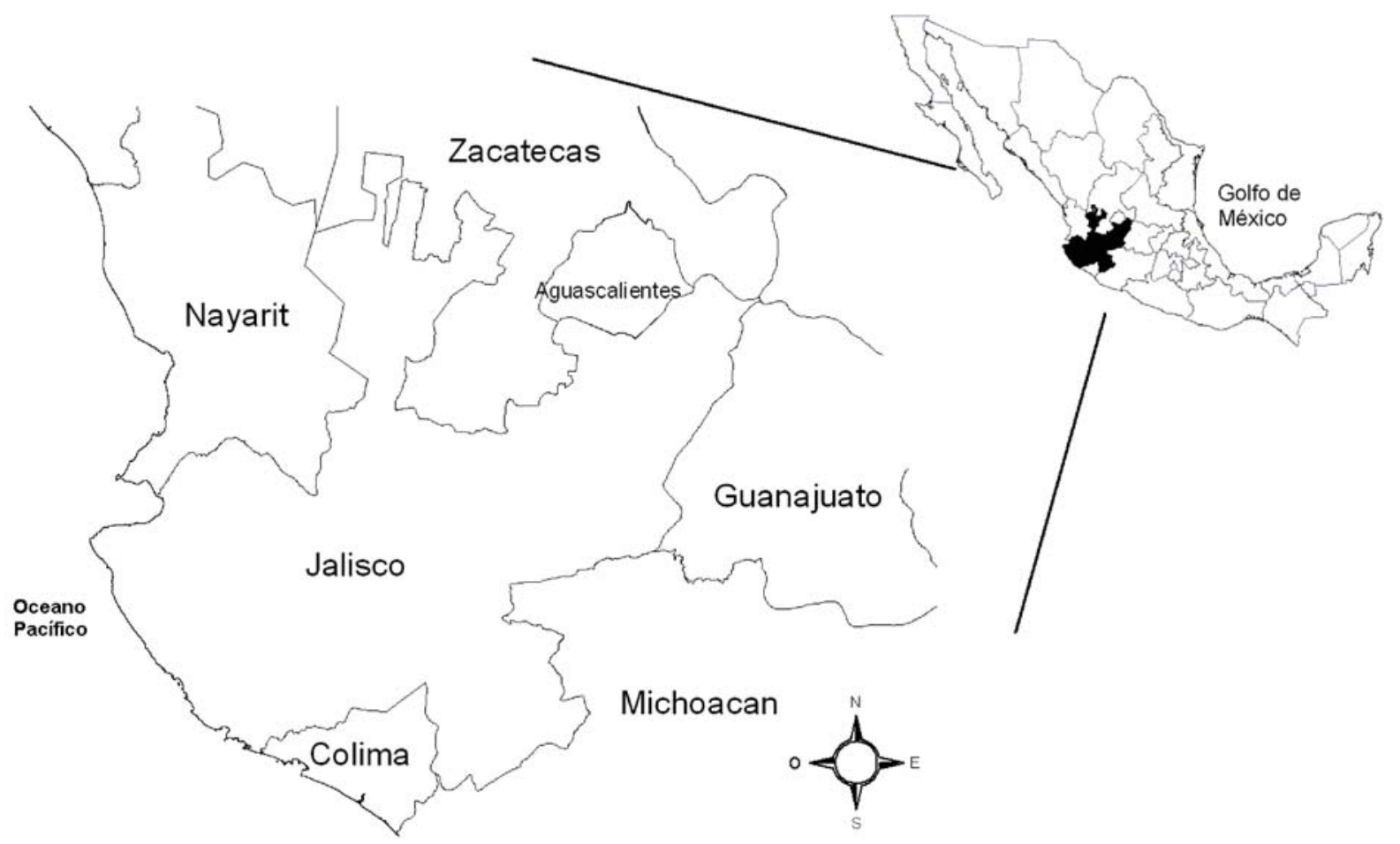

Figura 1. Ubicación geográfica del estado de Jalisco en la República Mexicana.

base de datos.

Colecciones nacionales consultadas. Colección de Vertebrados del Centro de Estudios en Zoología de la Universidad de Guadalajara (CZUG); Instituto de Biología de la Universidad Nacional Autónoma de México (CNMA); Facultad de Ciencias Biológicas de la Universidad Nacional Autónoma de Nuevo León (UANL), y el Instituto Manantlán de Ecología y Biodiversidad (IMECBIO).

Colecciones extranjeras consultadas: The Natural History Museum , University of Kansas (KU); Museum of Vertebrate Zoology, University of California (MVZ); The Museum, Texas Tech University (TTU); Texas Cooperative Wildlife Collection, Texas A \& M University (TCWC); American Museum of Natural History, New York (AMNH); California Academy of Sciences, Los Angeles (CAS); Mammal Collections, The Museum, East Lansing, Michigan State University (MSU); Field Museum of Natural History, Chicago (FMNH); Natural History Museum of the Smithsonian Institution (USNM), y The Natural History Museum of Los Angeles County (LACM).
Con base en la localidad de recolecta, cada registro se georreferenció en mapas de Jalisco escala 1:250,000. Se hizo una selección de los registros con base en los siguientes criterios: a) que presentaran localidad de recolecta completa; b) que la localidad de recolecta estuviera dentro de una UCG; $c$ ) nombre científico, y d) los registros de una misma especie con diferente localidad de recolecta (Kohlman y Sánchez-Colón, 1984; Prieto y Sánchez-Cordero, 1993; Sánchez, 1993; Murguía y Rojas, 2001; Illoldi et al., 2002). No se consideraron las especies introducidas e insulares y el arreglo taxonómico se basó en el trabajo de Guerrero y Cervantes (2003).

Se utilizó el paquete de computo ARC/INFO para generar una cobertura con la ubicación geográfica de los registros de mamíferos y para cambiar la proyección UTM a Lambert (Scott et al., 1993; Stow, 1993). La cobertura de puntos se sobrepuso con las UCG utilizando el programa de cómputo ARCVIEW 3.2. El procedimiento permitió conocer la presencia de especies en cada una de las UCG. De igual manera, la cobertura de puntos se sobrepuso a los mapas de vegetación y altitud que fueron obtenidos de la Comisión Nacional para el Conocimiento 


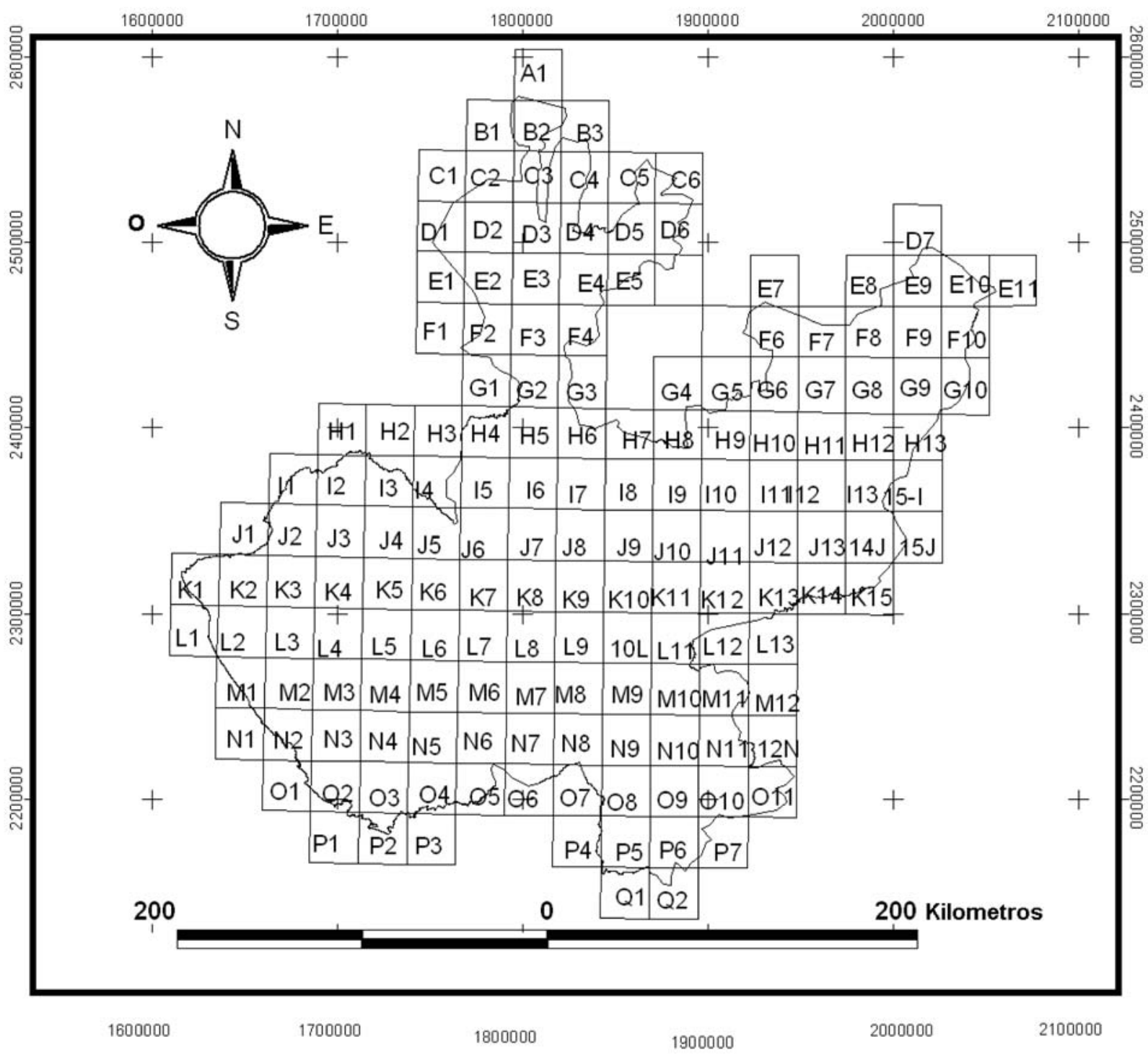

y Uso de la Biodiversidad (CONABIO) (Cuadro1), para integrar una matriz de presencia-ausencia de especies de mamíferos y analizar la distribución de especies de mamíferos por altitud y por tipo de vegetación. Para la altitud, se establecieron intervalos de $500 \mathrm{~m}$, desde cero hasta $4500 \mathrm{~m}$. Se utilizaron los factores de vegetación y altitud porque se ha demostrado que son los que influyen de forma determinante en la distribución de los mamíferos (Ceballos y Miranda, 1986; Fa y Morales, 1993; Kelt et al., 1999; Badgley y Fox, 2000; Bakker y Kelt, 2000; Williams et al., 2002).

Relación con factores del medio. Se realizó un análisis de ordenación de Bray-Curtis usando la transformación de suavización de Beals (Beals, 1984; McCune, 1994; McCune y Mefford, 1999; McCune y Grace, 2002) mediante varianza-regresión (ter Braak y Prentice, 1988) y el coeficiente de semejanza de Sorensen como medida de distancia. La ordenación se ejecutó mediante el programa de cómputo PC-ORD 4.10, ampliamente aceptado por la inclusión de diversas modificaciones que lo han convertido en un método valioso para la ordenación de datos ecológicos (Beals, 1984). Se le considera una de las técnicas más efectivas para representar gradientes ecológicos complejos (Beals, 1984; McCune y Beals, 1993; McCune y Grace, 2002). Las variables utilizadas en el análisis de ordenación se obtuvieron a partir de los mapas de CONABIO (Cuadro 1).

Como análisis complementario y con el objetivo de sustentar los grupos formados en la ordenación, se realizó un análisis de clasificación con la técnica UPGMA 
Cuadro 1. Nombre, referencia y unidad de medida de cada uno de los mapas obtenidos de la Comisión Nacional para el Conocimiento y Uso de la Biodiversidad (CONABIO).

\begin{tabular}{|c|c|c|}
\hline Nombre del mapa & Referencia bibliográfica & $\begin{array}{l}\text { Unidad de } \\
\text { medida }\end{array}$ \\
\hline Evotranspiración real & $\begin{array}{l}\text { Maderey, L. 1990. Evopotranspiración real en hidrogeografía IV.6.6. Atlas } \\
\text { Nacional de México. Vol. II Escala } 14000 \text { 000. Instituto de Geografía, } \\
\text { UNAM. México. }\end{array}$ & $\mathrm{mm}$ \\
\hline Hidrogeología & $\begin{array}{l}\text { Marín-C, S. y C. Torres-Ruata. 1990. Hidrogeología. IV. 6. 3. Atlas Nacional } \\
\text { de México. Vol. II Escala 1: } 4000 \text { 000. Instituto de Geografía, UNAM, } \\
\text { México. }\end{array}$ & \\
\hline $\begin{array}{l}\text { Precipitación total } \\
\text { anual }\end{array}$ & $\begin{array}{l}\text { García, E. y CONABIO. 1998. Precipitación total anual. Escala 1: } 1000000 . \\
\text { México. }\end{array}$ & $\mathrm{mm}$ \\
\hline $\begin{array}{l}\text { Provincias } \\
\text { biogeográficas de } \\
\text { México }\end{array}$ & $\begin{array}{l}\text { Comisión Nacional para el Conocimiento y Uso de la Biodiversidad } \\
\text { (CONABIO). 1997. Provincias biogeográficas de México. Escala 1:4 } 000000 . \\
\text { México. }\end{array}$ & \\
\hline $\begin{array}{l}\text { Regiones y provincias } \\
\text { mastogeográficas }\end{array}$ & $\begin{array}{l}\text { Ramírez-Pulido, J. y A. Castro-Campillo. 1990. Regiones y provincias } \\
\text { mastogeográficas en regionalización mastofaunística, IV.8.8. Atlas Nacional } \\
\text { de México. Vol. III. Escala 1:4 } 000 \text { 000. Instituto de Geografía, UNAM. } \\
\text { México. }\end{array}$ & \\
\hline $\begin{array}{l}\text { Temperatura media } \\
\text { anual }\end{array}$ & $\begin{array}{l}\text { Vidal-Zepeda, R. 1990. Temperatura media anual en temperatura media, } \\
\text { IV.4.4. Atlas Nacional de México. Vol. II. Escala 1:4 } 000 \text { 000. Instituto de } \\
\text { Geografía, UNAM. México. }\end{array}$ & ${ }^{\circ} \mathrm{C}$ \\
\hline $\begin{array}{l}\text { Temperaturas máxima } \\
\text { absoluta }\end{array}$ & $\begin{array}{l}\text { García, E. y CONABIO. 1998. Temperaturas máxima absoluta. Escala 1:1 } 000 \\
\text { 000. México. }\end{array}$ & ${ }^{\circ} \mathrm{C}$ \\
\hline $\begin{array}{l}\text { Temperaturas mínima } \\
\text { absoluta }\end{array}$ & $\begin{array}{l}\text { García, E. y CONABIO. 1998. Temperaturas mínima absoluta. Escala 1:1 } 000 \\
\text { 000. México. }\end{array}$ & ${ }^{\circ} \mathrm{C}$ \\
\hline $\begin{array}{l}\text { Topografía de } \\
\text { México, equidistancia } \\
\text { entre curvas } 200 \mathrm{~m}\end{array}$ & $\begin{array}{l}\text { Comisión Nacional para el Conocimiento y Uso de la Biodiversidad } \\
\text { (CONABIO). 1998. Topografía de México. Escala 1:250 000. Extraído del } \\
\text { Modelo Digital del Terreno. Instituto Nacional de Estadística, Geografía e } \\
\text { Informática (INEG). México. }\end{array}$ & msnm \\
\hline Vegetación potencial & $\begin{array}{l}\text { Rzedowski, J. 1990. Vegetación potencial. IV.8.2. Atlas Nacional de México. } \\
\text { Vol II. Escala1:4 } 000 \text { 000. Instituto de Geografía, UNAM. México. }\end{array}$ & \\
\hline
\end{tabular}

(Unweighted Pair-Group Method using arithmetic Average) usando como medida de distancia Jaccard, para lo cual se utilizó el programa Multivariate Statistical Package (MVSP 3.1). En virtud de que el análisis no mostró consistencia ni significancia biológica en sus resultados se optó por realizar la clasificación con TWINSPAN (Two Way Indicator Species Analysis) (McCune y Grace, 2002) mediante el programa PC-ORD 4.10 (McCune y Mefford, 1999).

\section{Resultados}

Patrones de distribución de la riqueza. Se obtuvieron 13938 registros de mamíferos para Jalisco en los cuales están representadas 168 especies de mamíferos. De éstos, 13537 fueron georreferenciados y 384 se excluyeron por no cumplir con los requisitos establecidos. El $23.5 \%$ de los registros excluidos no tenían la localidad de recolecta completa, el $16.4 \%$ no tenían el nombre científico, el $11.4 \%$ eran registros de una especie con la misma localidad de recolecta y el $48.6 \%$ eran especies introducidas. Los órdenes con mayor número de registros fueron Rodentia (54\%) y Chiroptera (41\%). En contraste, Cingulata (0.25\%) presentó el menor número de registros. La familia con la mayor cantidad de registros fue Muridae (34\%) seguida de Phyllostomydae (26\%) y Heteromydae (13\%); para las restantes familias se registraron valores menores a $4 \%$ cada una. El género mejor representado fue Peromyscus mientras que el de menos registros fue Choeroniscus. La 
especie con mayor número de registros fue Liomys pictus, en tanto que Reithrodontomys zacatecae y Nyctinomops macrotis sólo tuvieron un registro.

De las 159 UCG, 39 no tuvieron registros $(24.5 \%)$ y 120 tuvieron, al menos, uno, lo cual representa el $75.5 \%$. El $8.3 \%$ de las UCG tienen más de 40 especies, mientras que el $34.1 \%$ de las UCG presentaron menos de 10 especies. Las UCG con mayor número de especies fueron San Cristóbal de la Barranca (H7), la Estación de Biología Chamela (N2), el Nevado de Colima (N8), la Estación Científica de Las Joyas (N5), Tequila (H6), El Grullo (M6) y Talpa de Allende (L4, K4) (Fig. 3). El promedio de especies por UCG fue de $18 \pm 13.9$.

En el análisis de diversidad por intervalos de altitud, se observaron 128 especies $(76.1 \%$ ) entre los 1500 y $2000 \mathrm{~m}$. Le siguieron los intervalos 1001 y $1500 \mathrm{~m}$ con 127 especies $(75.5 \%)$ y 2001 a 2500 m con 124 especies (73.8\%). En los intervalos de altitud 0-500 m y 501-1000 $\mathrm{m}$, se registró una diversidad media representada por 110 y 103 especies respectivamente. Estas observaciones muestran una tendencia a disminuir hacia las partes de mayor altitud, sobre todo, después de los 2500 m donde se aprecia un declive en la diversidad (Fig. 4).

El análisis de diversidad por tipo de vegetación mostró que en el bosque tropical caducifolio se registraron 154 especies (91.6\%) y en el bosque de coníferas y encinos 150 especies $(89.2 \%)$, mientras que el tipo de vegetación con menos especies fue el bosque espinoso con 12 especies $(9.5 \%)$ (Fig. 5).

Relaciones con los factores del medio. El primer eje de la ordenación explicó el $84.9 \%$ de la variación; el segundo explicó el $7.31 \%$ con un valor acumulado del $92.22 \%$; y el tercero, el $1.78 \%$ con un valor acumulado del $94 \%$. El primer eje resultó correlacionado positivamente con variables climáticas como promedio de evaporación (Pevapo) $(\mathrm{r}=0.796)$, promedio de temperatura mínima (Ptemmin) $(\mathrm{r}=0.754)$, promedio de temperatura (Ptempe) $(\mathrm{r}=0.739)$, total de precipitación (Tprecipi) $(\mathrm{r}=0.715)$,

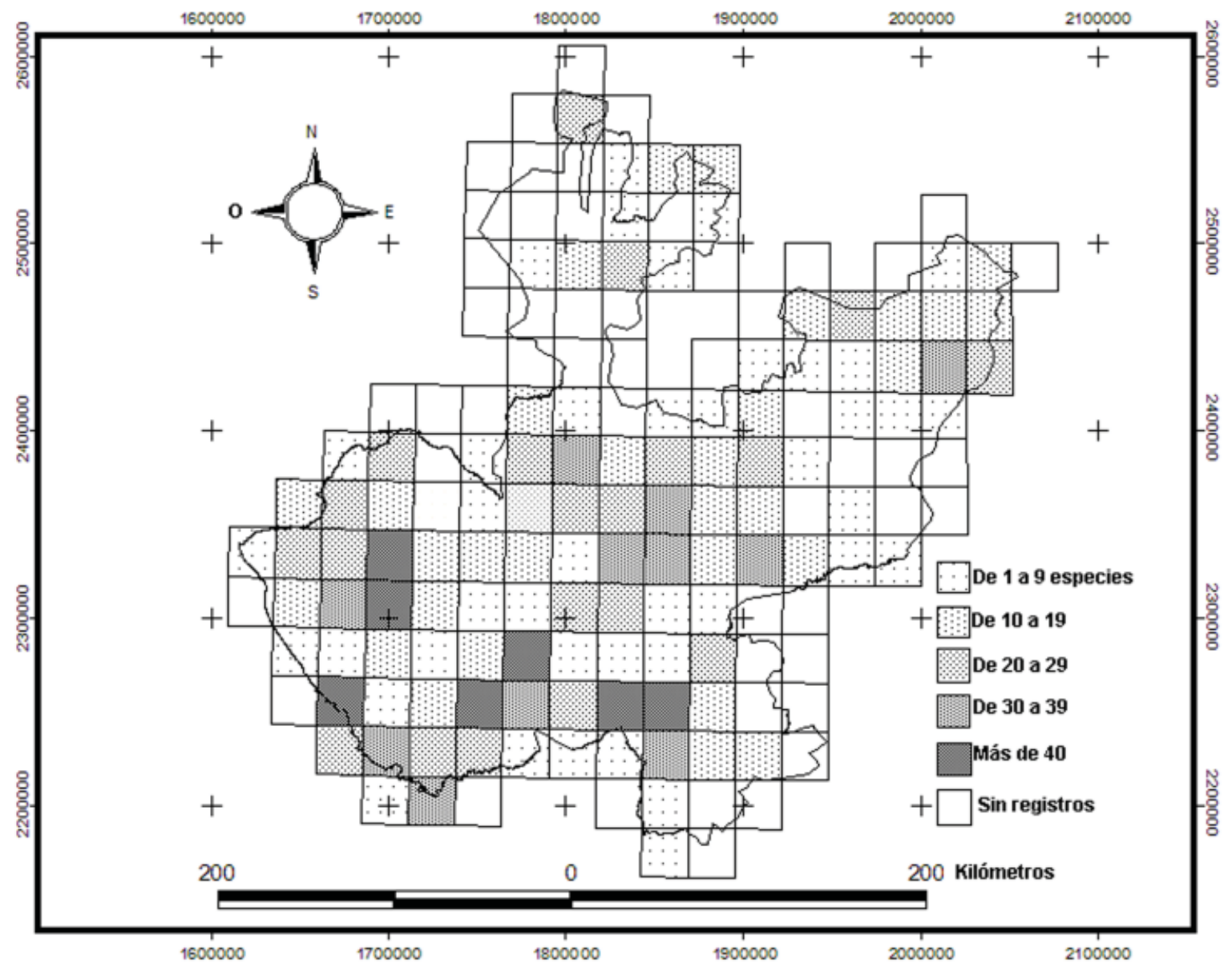

Figura 3. Distribución de la riqueza de especies respecto a las unidades de clasificación geográfica (UGC) con base en 13938 registros del estado de Jalisco. El sistema de coordenadas utilizado es UTM. 


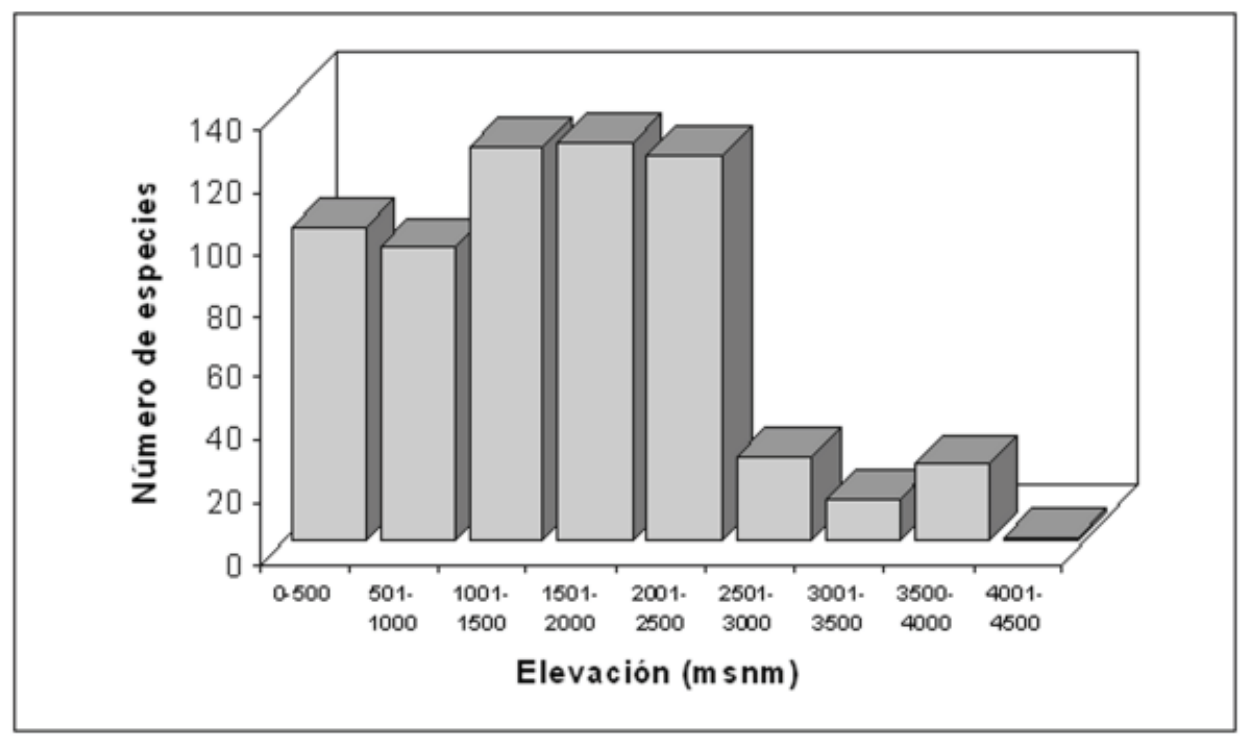

Figura 4. Distribución de la riqueza de especies de mamíferos por intervalos de elevación (msnm).

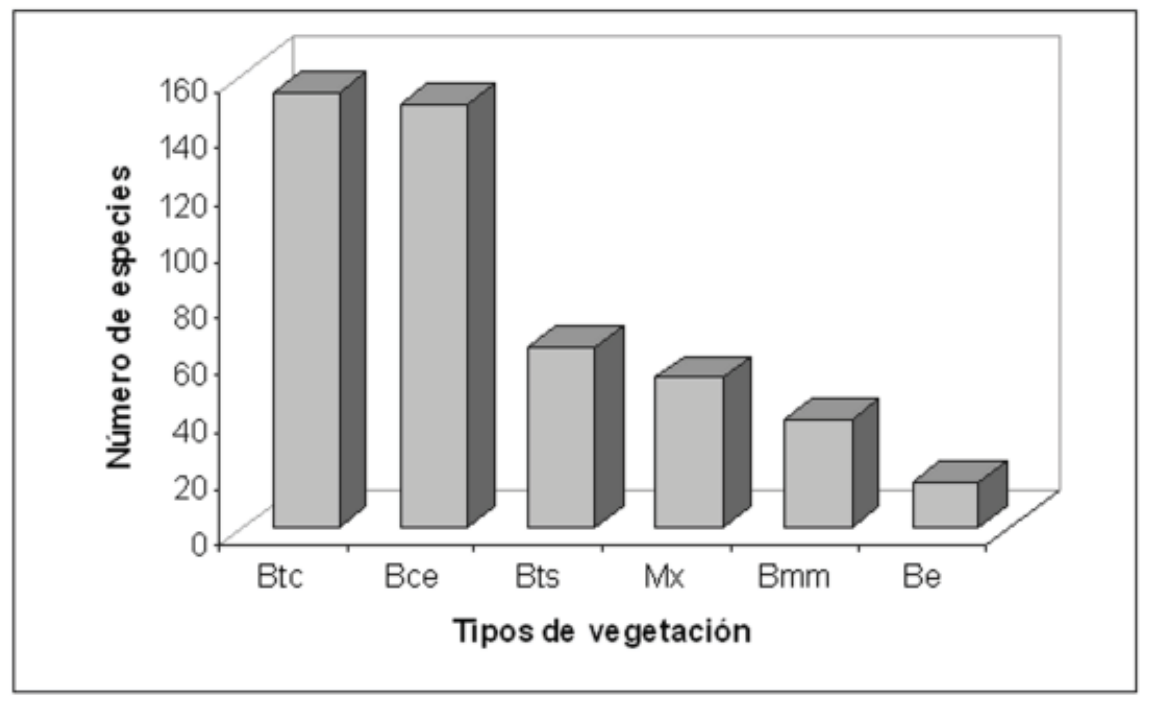

Figura 5. Distribución de la riqueza de especies por tipo de vegetación con base en la clasificación de Rzedowski (1986). Btc = bosque tropical caducifolio, $\mathrm{Bce}=$ bosque de coníferas y encinos, $\mathrm{Bts}=$ bosque tropical subcaducifolio, $\mathrm{Mx}=$ matorral xerófilo, $\mathrm{Bmm}=$ bosque mesófilo de montaña y $\mathrm{Be}=$ bosque espinoso.

coeficiente de variación de la altitud (CValtit) $(\mathrm{r}=0.604)$, promedio de temperatura máxima (Ptemmax) $(\mathrm{r}=0.506)$; y negativamente con promedio de altitud (Paltitud) $(\mathrm{r}=$ -0.707) y tipo de vegetación (Tvegeta) $(r=-0.534)$ (Fig. 6; Cuadro 2).

Los grupos formados por el análisis de clasificación TWINSPAN fueron corroborados por la ordenación (Figs. 6, 7 y 8). El grupo A está formado por especies de mamíferos que se distribuyen al suroeste en la región de la costa y parte adyacente al Eje Neovolcánico, se caracterizó por presentar una variación altitudinal que va de 0 a 2200 $\mathrm{m}$, con un promedio de temperatura mínima mayor de $10.63^{\circ} \mathrm{C}$ y una precipitación promedio de $1174.48 \mathrm{~mm}$; las especies indicadoras fueron Osgoodomys banderanus y Artibeus phaeotis (Cuadro 3).

El grupo B está representado por las siguientes 


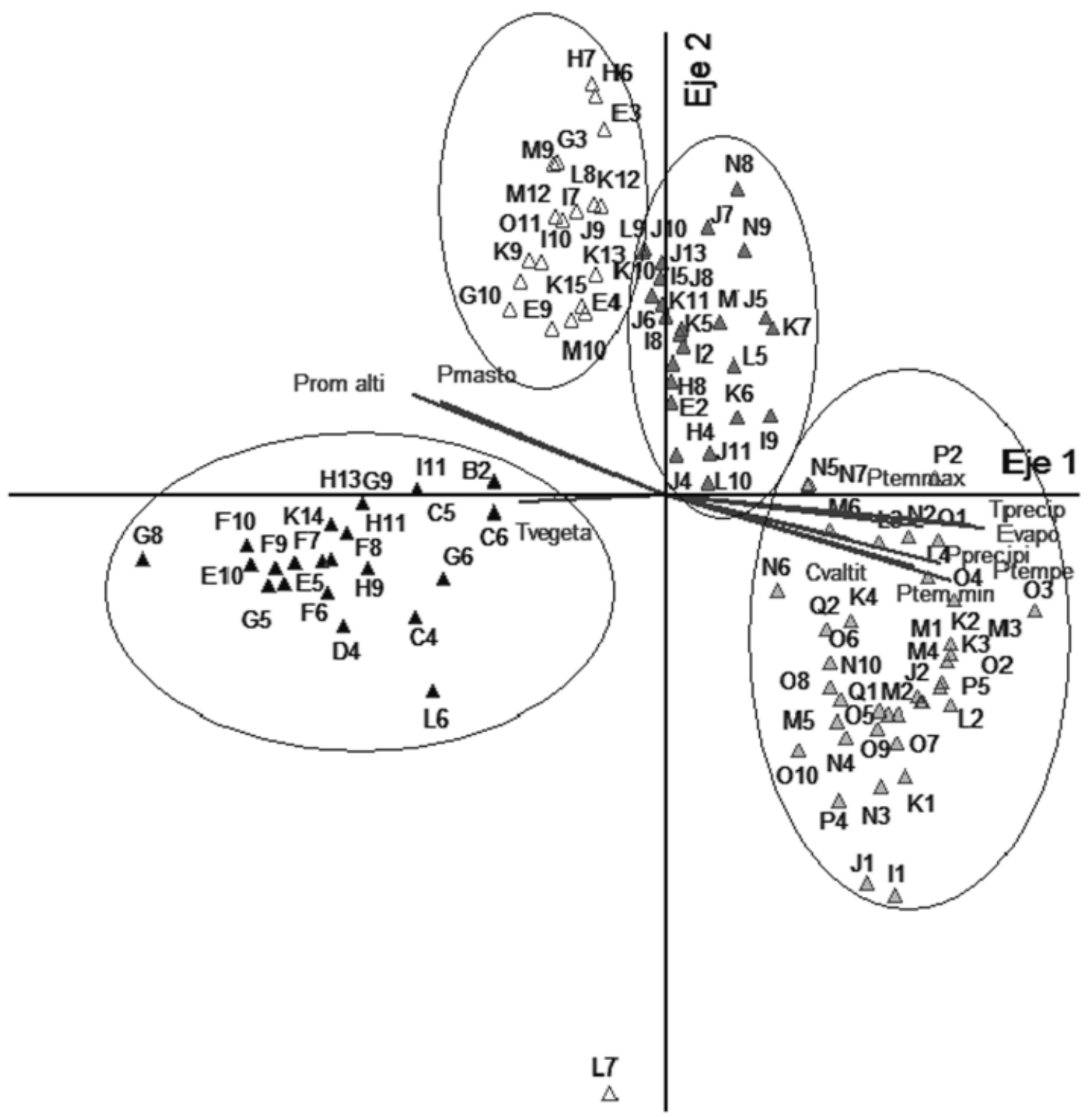

Figura 6. Representación gráfica del análisis de ordenación Bray-Curtis utilizando el programa de cómputo PcOrd, para 108 UCG en el estado de Jalisco y 168 especies. Se muestra el primer eje con el segundo y los grupos de mamíferos observados. Los vectores representan variables del medio y tienen una correlación mayor a 0.35. Donde Cvalti= coeficiente de variación, Evapo= evapotranspiración real, Pmasto $=$ provincia mastozoológica, Ptemmax $=$ promedio de temperatura máxima, Pprecipi $=$ promedio de precipitación, Pro alti $=$ promedio de altitud, Ptemmin $=$ promedio de temperatura mínima, Ptempe $=$ promedio de temperatura, Tprecip= precipitación total y Tvegeta $=$ tipo de vegetación.

especies: Liomys irroratus, Peromyscus maniculatus y Baiomys taylori, que se distribuyen en la zona central del estado. Se caracterizó por presentar un promedio de altitud de $1802.78 \mathrm{~m}$, un promedio de temperatura mínima de $5.47^{\circ} \mathrm{C}$ y una precipitación promedio de $903.33 \mathrm{~mm}$.

En el grupo C las especies indicadoras fueron: Neotoma mexicana y Peromyscus spicilegus, que se distribuyen en la zona central de Jalisco. Se caracterizó por presentar un promedio de altitud de $1744.67 \mathrm{~m}$, un promedio de temperatura mínima de $5.84^{\circ} \mathrm{C}$ y una precipitación promedio de $845.14 \mathrm{~mm}$.

El grupo D corresponde a las UCG del noreste del estado en la meseta central y se caracteriza por tener una menor variación altitudinal que va de 1700 a 2100 
Cuadro 2. Valores de correlación de las variables con los ejes de ordenación, obtenidos a partir del análisis de ordenación BrayCurtis. Los números en negritas indican que son estadísticamente significativos $(\mathrm{p}<0.05)$.

\begin{tabular}{|c|c|c|c|}
\hline Eje & 1 & 2 & 3 \\
\hline Variable & $r$ & $r$ & $\mathrm{r}$ \\
\hline $\begin{array}{l}\text { Promedio de } \\
\text { evapotranspiración }\end{array}$ & 0.796 & -0.256 & -0.356 \\
\hline $\begin{array}{l}\text { Evapotranspiración } \\
\text { real }\end{array}$ & 0.779 & -0.244 & -0.347 \\
\hline $\begin{array}{l}\text { Promedio de } \\
\text { temperatura mínima }\end{array}$ & 0.754 & -0.411 & -0.462 \\
\hline $\begin{array}{l}\text { Promedio de } \\
\text { temperatura }\end{array}$ & 0.739 & -0.367 & -0.422 \\
\hline $\begin{array}{l}\text { Precipitación total } \\
\text { anual }\end{array}$ & 0.715 & -0.253 & -0.376 \\
\hline $\begin{array}{l}\text { Promedio de } \\
\text { precipitación }\end{array}$ & 0.702 & -0.377 & -0.477 \\
\hline $\begin{array}{l}\text { Coeficiente de } \\
\text { variación de la altitud }\end{array}$ & 0.604 & -0.298 & -0.396 \\
\hline $\begin{array}{l}\text { Promedio de } \\
\text { temperatura máxima }\end{array}$ & 0.506 & -0.176 & -0.238 \\
\hline Tipo de geología & 0.400 & -0.338 & -0.366 \\
\hline $\begin{array}{l}\text { Heterogeneidad de } \\
\text { temperatura mínima }\end{array}$ & 0.347 & 0.257 & 0.176 \\
\hline $\begin{array}{l}\text { Provincia } \\
\text { biogeográfica }\end{array}$ & 0.100 & 0.366 & 0.341 \\
\hline Tipo de vegetación & -0.534 & 0.120 & -0.011 \\
\hline $\begin{array}{l}\text { Provincia } \\
\text { mastozoológica }\end{array}$ & -0.666 & 0.430 & 0.521 \\
\hline Promedio de altitud & -0.707 & 0.446 & 0.471 \\
\hline
\end{tabular}

$\mathrm{m}$, con promedio de temperaturas mínimas de $3.55^{\circ} \mathrm{C}$, y precipitación promedio de $727.08 \mathrm{~mm}$; las especies indicadoras fueron: Neotoma leucodon y Peromyscus gratus.

El grupo A se asocia con el B y el C con el D (Fig. 7). Estas asociaciones se diferencian claramente una de la otra, mientras que los grupos $\mathrm{B}$ y $\mathrm{C}$ se distribuyen de forma dispersa en la parte central del estado (Figs. 6, 7 y 8).

\section{Discusión}

Patrones de distribución de la riqueza. De acuerdo con los resultados obtenidos, se observa una alta heterogeneidad en la distribución del número de registros de mamíferos para el estado de Jalisco. Se pueden encontrar tanto las
UCG que tienen una alta riqueza de especies, como las de baja riqueza e incluso con ausencia de especies.

La baja riqueza observada en algunas de las UCG de la zona costera de Jalisco puede explicarse por la menor área de territorio que contienen, mientras que no sucede lo mismo con las que se encuentran alrededor de la entidad, ya que se tomó como zona de estudio la UCG completa. Las UCG en las que se observó la mayor riqueza contrastaron con aquellas que se encuentran a su alrededor, lo que puede interpretarse como un mayor esfuerzo de recolecta en ciertas zonas del estado y en especial en las UCG donde se localizan áreas con alguna categoría de protección. Dada la elevada cantidad de registros integrados en el presente estudio, los cuales representan más del $90 \%$ de los depositados en colecciones de Estados Unidos y Canadá para Jalisco (López-Wilchis et al., 1998), se puede decir que las UCG sin registros de mamíferos no han sido exploradas, por lo que valdría la pena encauzar esfuerzos para obtener la información pertinente. Estas mismas UCG sin registros coinciden con las señaladas por Maraña-Peña (1999) para carnívoros y con López (2001) para múridos, lo que significa que una tercera parte del estado no tiene información de registros para la clase Mammalia. A pesar de la heterogeneidad observada en las UCG se pudieron detectar patrones de distribución generales, ya que según se ha registrado el esfuerzo de muestreo, incluso cuando no es equitativo en todas las áreas, no oscurece completamente los patrones geográficos (O’Hara y Poore, 2000; Lobo et al., 2001; Lobo y Martín-Piera, 2002).

Respecto a los sitios con mayor riqueza de especies, sobresalen las UCG de la Estación de Biología Chamela y la Estación Científica de Las Joyas. En dichas UCG es factible que la riqueza observada se deba a un mayor esfuerzo de recolecta y a una mayor cantidad de estudios en la zona, por tratarse ambas de áreas naturales protegidas con presencia de una institución académica que apoya su manejo. En contraste, hay que resaltar la riqueza de especies observada en el Nevado de Colima, que si bien tiene la categoría de Parque Nacional ha sido objeto de menos estudios que las anteriores (Ceballos et al., 2002a). Esta riqueza de especies en el Nevado de Colima coincide con lo que López (2001) registra para múridos, mientras que la mayor riqueza de especies en la Estación de Chamela coincide con el trabajo de Maraña-Peña (1999).

Es relevante haber encontrado en el estado zonas que presentan una alta diversidad de especies y, sin embargo, no tienen categoría alguna de protección biológica , como San Cristóbal de la Barranca (H7), Tequila (H6), El Grullo (M6) y Talpa de Allende (L4, K4).

La distribución de la riqueza de especies de mamíferos en Jalisco presenta un gradiente que va de la región tropical a la zona semiseca, en donde la zona noreste presenta una 


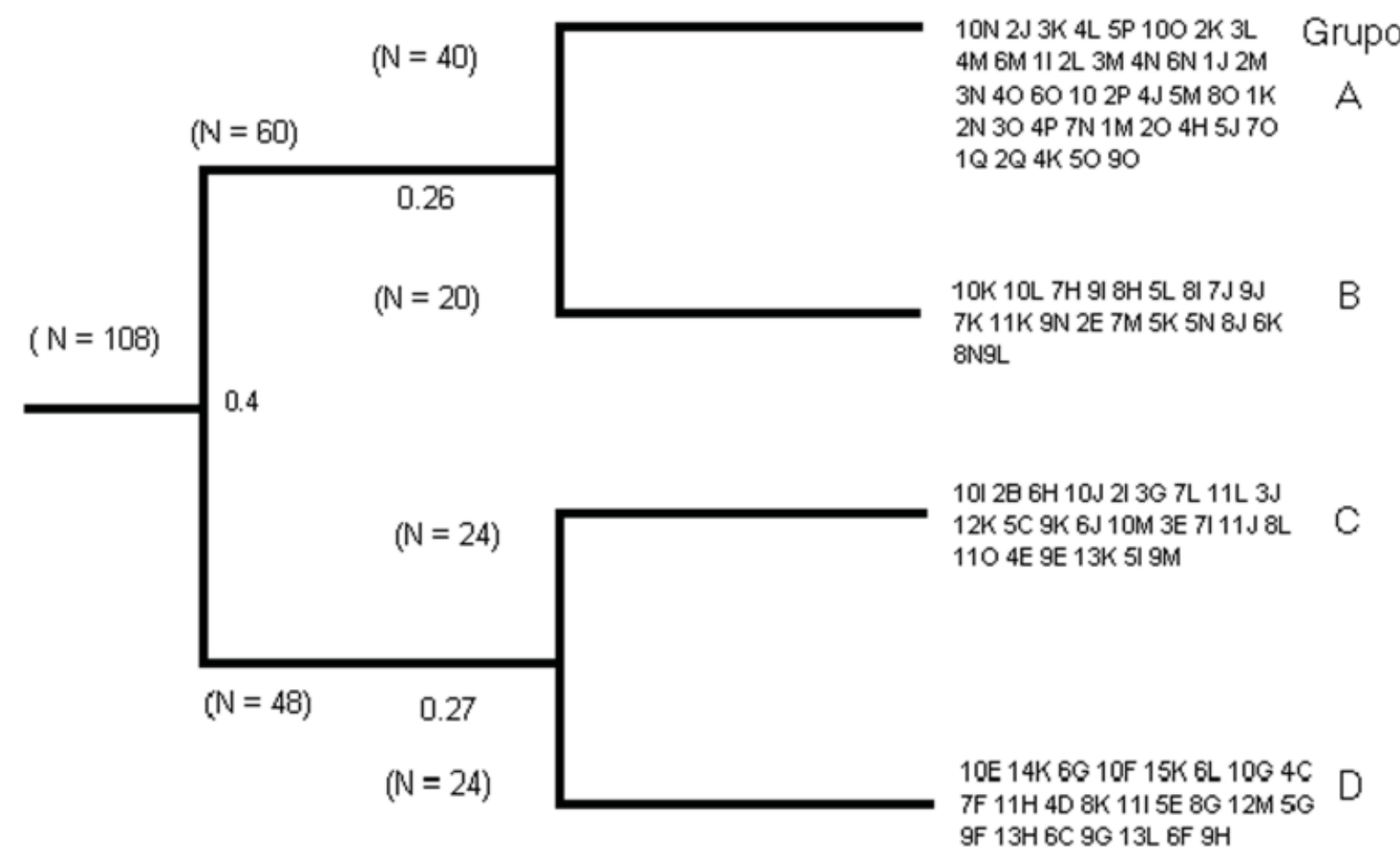

Figura 7 Dendrograma derivado del análisis de clasificación TWINSPAN, con 168 especies de mamíferos en el estado de Jalisco y 108 UCG. Grupo A (mamíferos de la costa), grupo B (mamíferos del centro a), grupo C (mamíferos del centro b), y grupo D (mamíferos del noreste).

menor riqueza en comparación con la región de la costa. Esto concuerda con lo que informan Fa y Morales (1993), Guerrero et al. (1995), Ceballos y García (1995), Ceballos et al. (1998), Maraña-Peña (1999) y Ceballos et al. (2002 a).

De acuerdo con el análisis de la riqueza de mamíferos por tipos de vegetación, se observó que el bosque tropical caducifolio y el bosque de coníferas y encinos fueron los que presentaron un mayor número de especies. El bosque tropical caducifolio, tal como lo señalan Badgley y Fox (2000), Ceballos y García (1995) y Fa y Morales (1993) contiene una alta riqueza de mamíferos. Una parte de la riqueza del bosque tropical caducifolio y del bosque de coníferas y encino, se puede explicar por el hecho de que éstos son los tipos de vegetación que mayor superficie ocupan en el estado (INEGI, 1981). Además, la riqueza en el bosque tropical caducifolio está relacionada con una mayor heterogeneidad estructural en la vegetación (Deacon et al., 1964; Graham, 1983; Owen, 1990; Bakker y Kelt, 2000; Lomolino, 2001), en donde su estratificación con respecto a otros tipos de vegetación conlleva a un aumento en la diversidad (Bakker y Kelt, 2000), lo cual es una de las explicaciones al gradiente latitudinal en la riqueza de especies de mamíferos como observan Kaufman (1995), Kaufman y Willig (1998) y Ceballos et al. (2002a).

Relaciones con los factores del medio. Con base en los resultados del análisis de ordenación, las variables que explican la distribución de los mamíferos en Jalisco fueron temperatura promedio máxima y mínima, altitud promedio y su coeficiente de variación, evaporación, precipitación y vegetación. Badgley y Fox (2000), Currie (1991), Illoldi et al. (2002), Matson (1982) y Owen (1990) recalcan que la temperatura y altitud son factores determinantes en la distribución de los mamíferos. Aunque también se ha observado que variables como un periodo libre de heladas, latitud, humedad, evaporación, aridez, vegetación, precipitación y heterogeneidad del hábitat tienen una marcada influencia en la definición de los patrones que presentan los mamíferos en su distribución regional (Matson,1982; Owen, 1990; Fa y Morales, 1993; Kelt et al., 1999; Badgley y Fox, 2000; Bakker y Kelt, 2000; Lomolino, 2001; Virgós et al., 2001; Illoldi et al., 2002; Hawkins et al., 2003).

De manera general se puede señalar que la distribución de los mamíferos en el estado de Jalisco está influenciada por un gradiente climático, donde destaca la temperatura 


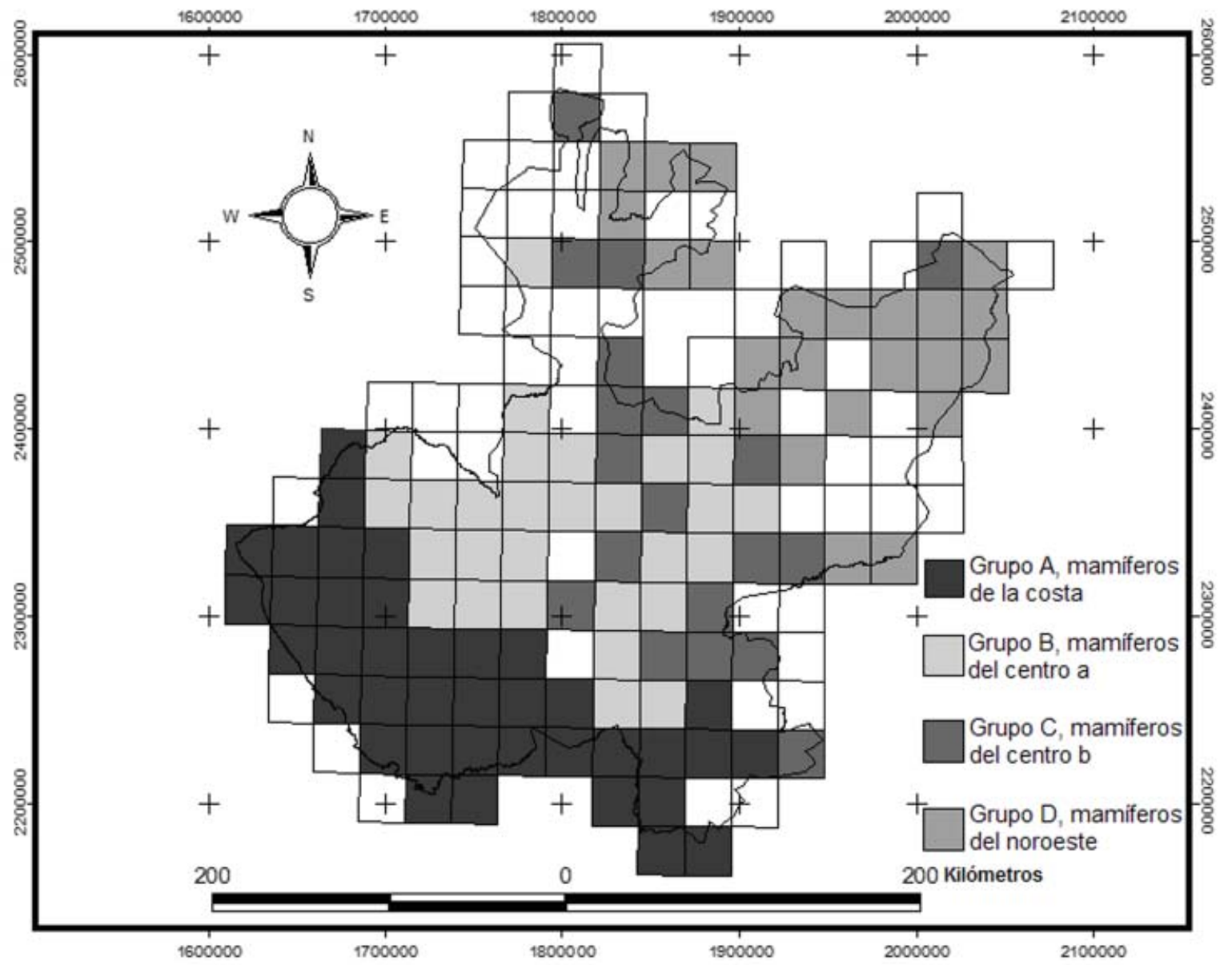

Figura 8. Mapa derivado del análisis de ordenación y clasificación TWINSPAN, con 168 especies de mamíferos en el Estado de Jalisco y 108 UCG. Grupo A ( mamíferos de la costa), grupo B (mamíferos del centro a), grupo C (mamíferos del centro b), y grupo D (mamíferos del noreste). El sistema de coordenadas utilizado es UTM.

y la disponibilidad de agua, lo que concuerda con lo que Badgley y Fox (2000) encontraron para los mamíferos de Norteamérica y Hawkins et al. (2003) para plantas, vertebrados e invertebrados. Los últimos registran la existencia de un gradiente diferencial en la importancia de las variables agua-energía en la explicación de la distribución de las especies, en donde a latitudes cercanas al ecuador la disponibilidad de agua es el factor que mejor explica la distribución de especies, mientras que para latitudes altas es la energía, y para las intermedias, la combinación de ambas, lo cual concuerda con los resultados obtenidos en Jalisco. Esta dinámica entre agua y energía sugiere que dichas variables tienen un papel importante en la fisiología de los mamíferos. Se da por hecho que la reproducción y el desarrollo de los mamíferos es mayor en zonas con temperaturas promedio altas porque es menor el costo metabólico para la termorregulación, por lo cual la precipitación presenta mayor relevancia en la distribución de los mamíferos. Es evidente que en zonas semiáridas y desérticas la disponibilidad de agua sea un factor de más importancia. Por el contrario, en zonas con temperaturas promedio bajas, la variable con mayor peso es la energía en vez de la disponibilidad de agua (Currie, 1991; Hawkins et al., 2003).

Se observan 2 grupos claramente diferenciados: el primero corresponde a las especies de la costa, zona que se caracteriza por una mayor precipitación pluvial, mayor evaporación, promedio alto de temperatura y temperatura mínima alta, una marcada variación de la altitud que va desde los 0 hasta los $1500 \mathrm{~m}$, un clima de tipo cálidotemplado, y la presencia de bosque tropical caducifolio y subcaducifolio, bosque de coníferas y encinos y bosque mesófilo de montaña; el segundo está integrado por especies que habitan la región semiárida del noreste del estado, la cual se caracteriza por tener una baja precipitación pluvial y evaporación, temperaturas mínimas menores a $10^{\circ} \mathrm{C}$, la 
Cuadro 3. Características ambientales de los grupos de las UCG derivados del análisis de ordenación y clasificación TWINSPAN (promedio \pm desviación estándar).

\begin{tabular}{lllll}
\hline & Grupo A & Grupo B & Grupo C & Grupo D \\
\hline $\begin{array}{l}\text { Evaporación }(\mathrm{mm}) \\
\text { Altitud (msnm) }\end{array}$ & $848.75 \pm 102.21$ & $736.67 \pm 50.02$ & $702.08 \pm 117.47$ & $581.25 \pm 83.16$ \\
$\begin{array}{l}\text { Temperatura } \\
\left({ }^{\circ} \mathrm{C}\right)\end{array}$ & $23.29 \pm 2.08$ & $19.61 \pm 1.63$ & $19.80 \pm 1.68$ & $18.06 \pm 1.1$ \\
$\begin{array}{l}\text { Precipitación } \\
(\mathrm{mm})\end{array}$ & $1174.48 \pm 290.39$ & $903.33 \pm 130.49$ & $845.14 \pm 170.26$ & $727.08 \pm 158.78$ \\
$\begin{array}{l}\text { Temperatura } \\
\text { máxima }\left({ }^{\circ} \mathrm{C}\right)\end{array}$ & $33.33 \pm 1.37$ & $31.84 \pm 2.37$ & $31.67 \pm 2.14$ & $30.44 \pm 1.43$ \\
$\begin{array}{l}\text { Temperatura } \\
\text { mínima }\left({ }^{\circ} \mathrm{C}\right)\end{array}$ & $10.63 \pm 2.95$ & $5.47 \pm 1.51$ & $5.84 \pm 2.09$ & $3.55 \pm 1.11$ \\
\hline
\end{tabular}

presencia de vegetación xerófila y clima seco.

Entre estos 2 grupos claramente definidos, en la parte central del estado y en la zona norte, que corresponde a la región de la Sierra Madre Occidental, parecen encontrarse otros 2, que no se logran diferenciar adecuadamente.

Lo anterior, en principio, puede deberse a que en la región central tenemos un mosaico de variables ambientales y diferentes condiciones topográficas, es decir, hay una gran heterogeneidad (INEGI, 1981) que permite encontrar mastofauna que se distribuye tanto en la zona tropical como en la árida y la confluencia de estas especies en el centro del estado no facilita observar un patrón claro de distribución.

Una segunda explicación puede ser la evidencia de un patrón de riqueza diferente entre los mamíferos no voladores y los murciélagos (Fleming, 1973; Guerrero et al., 1995; Maraña-Peña, 1999; Badgley y Fox, 2000; López, 2001; Ceballos et al., 2002a), en el cual estos últimos presentan una mayor riqueza de especies hacia las zonas tropicales respecto de las zonas áridas y semiáridas, y los roedores presentan un patrón inverso. Estos 2 patrones de distribución de la riqueza se pueden estar traslapando en la parte central del estado. Por lo tanto, al considerar grupos de mamíferos por separado puede surgir un patrón diferente al que se obtiene cuando se considera toda la clase. Esto resulta importante si tomamos en cuenta que el $94.4 \%$ de los registros para el presente trabajo fueron de quirópteros y roedores. A pesar de estas evidencias de un patrón inverso entre los murciélagos y los mamíferos no voladores, algunos autores (Kaufman, 1995; Kaufman y Willig, 1998 y Ortega y Arita, 1998) enfatizan la importancia de incluir ambos grupos en la descripción de patrones de distribución, dado que de manera conjunta contribuyen a dicho patrón, razón por la que en el presente estudio fueron analizados de esta forma.

Una tercera explicación a lo observado puede radicar en la naturaleza de los datos y en la presencia de UCG carentes de registros de mamíferos. De tal forma que si se hiciera un análisis por grupos y se aumentase el número de registros para la zona, se esperaría que el centro y norte del estado conformaran un solo grupo o posiblemente que se separaran en 2 distintos grupos, pero sin afectar el patrón general, lo cual ya se observó en estudios previos (Ceballos y Rodríguez, 1993; Fa y Morales, 1993; Guerrero et al., 1995; Ceballos et al., 1998; Ceballos et al., 2002 a; Escalante et al., 2002).

El patrón en la distribución de la riqueza observado en el estado de Jalisco puede contribuir en buena medida al establecimiento de áreas naturales protegidas (Scott et al., 1993; Palomera-García et al., 1994; Ceballos et al., 1998; Escalante et al., 2002), ya que si aceptamos que la distribución de un organismo no obedece al azar sino a la conjunción de factores ambientales, ecológicos e históricos, muchos de los procesos que han operado y operan actualmente no sólo afectan a un grupo de organismos, sino que probablemente afecten a muchos otros que pueden exhibir patrones semejantes (O'Hara y Poore, 2000; Escalante et al., 2002). Con base en estos patrones, aquellas zonas que albergan la mayor riqueza específica pueden considerarse como las que pueden estar sujetas a algún tipo de protección (Scott et al., 1993; Palomera-García et al., 1994), como en los casos del cerro de Tequila, San Cristóbal de la Barranca, Tapalpa y El Grullo. En este sentido, resalta que 3 de las UCG donde se 
ubicaron áreas naturales con alguna categoría de protección biológica (el Parque Nacional Nevado de Colima, la Estación de Biología Chamela y la Estación Científica de Las Joyas) fueron de las que mayor número de especies de mamíferos presentaron. Además de éstas, hay otras áreas naturales propuestas para ser protegidas, como Cajón de Peñas (31 especies), San Sebastián del Oeste (18 especies) y Sierra San Isidro Cuatralba (19 especies) que registraron una riqueza importante de mamíferos, aunado a que las 2 primeras se encuentran dentro de las áreas terrestres prioritarias para la conservación en México, detectadas por Ceballos et al. (1998) y establecidas por CONABIO. No deben olvidarse las especies endémicas, que no siempre coinciden con las regiones de mayor riqueza, como es el caso de los mamíferos (Ceballos y Rodríguez 1993; Fa y Morales, 1993; Ceballos y Brown, 1995; Guerrero et al., 1995; Villaseñor et al., 2003).

Con base en nuestros resultados se observa que la fauna de mamíferos que se distribuye en la zona noreste de Jalisco presenta características que la diferencian de las demás áreas. El estado de Jalisco no tiene representación de esta fauna en un área protegida, por lo que sería conveniente considerar la zona noreste como prioritaria para la investigación, con lo cual se establecerían las bases para la conservación y representación de los taxa y la riqueza de la mastofauna característica de dicha región.

Otra de las ventajas en el estudio de patrones de distribución por el método utilizado aquí es poder identificar, como áreas prioritarias para la investigación aquellas en las que aún no se ha trabajado suficientemente (O'Hara y Poore, 2000; Escalante et al., 2002), entre las que se pueden mencionar la parte norte de Jalisco, que corresponde a la Sierra Madre Occidental; la parte sureste, que corresponde a la Sierra Madre del Sur y las de algunos municipios, como Arandas, Jesús María y Ayotlán que presentaron varias UCG sin registro de mamíferos.

\section{Literatura citada}

Badgley, C. y D. Fox. 2000. Ecological biogeography of North American mammals: species density and ecological structure in relation to environmental gradients. Journal of Biogeography 27:1437-1467.

Bakker, V. y D. Kelt. 2000. Scale-dependent patterns in body size distributions of neotropical mammals. Ecology 81:3530-3547.

Beals, E. 1984. Bray-Curtis ordination: an effective strategy for analysis of multivariate ecological data. Advances in Ecological Research: 1-55.

Ceballos, G., J. Arroyo-Cabrales y R. Medellín. 2002a. Mamíferos de México. In: Diversidad y conservación de los mamíferos neotropicales, G. Ceballos y J. Simonetti (eds.). CONABIO y Universidad Nacional Autónoma de México, México, D. F. p. 377-413.

Ceballos, G., J. Arroyo-Cabráles y R. Medellín. 2002b. The mammals of México: composition distribution, and conservation status. Occasional Papers The Museum of Texas Tech University 218:1-27.

Ceballos, G. y A. García. 1995. Conserving neotropical biodiversity: The role of dry forests in western México. Conservation Biology 9: 1349-1356.

Ceballos, G. y A. Miranda. 1986. Los mamíferos de Chamela, Jalisco. Instituto de Biología, Universidad Nacional Autónoma de México, México, D.F. 667 p.

Ceballos, G. y D. Navarro. 1991. Diversity and conservation of Mexican mammals. In Latin American mammalogy: history, biodiversity, and conservation, M. Mares, D. Schmidly (eds.). University of Oklahoma Press, Norman. p. 167-98.

Ceballos, G. y P. Rodríguez. 1993. Diversidad y conservación de los mamíferos de México: II Patrones de endemicidad. In Avances en el estudio de los mamíferos de México, Publicaciones Especiales, vol. 1, R. Medellín y G. Ceballos (eds.). Asociación Mexicana de Mastozoología, México, D. F. p. 86-108.

Ceballos, G., P. Rodríguez y A. Medellín. 1998. Assessing conservation priorities in megadiverse México: Mammalian diversity, endemicity, and endangerment. Ecological Applications 8: 8-17.

Collins, S. y S. Glenn. 1997. Effects of organismal and distance scaling on analysis of species distribution and abundance. Ecological applications 7: 543-551.

Currie, D. 1991. Energy and large-scale patterns of animaland plant-species richness. The American Naturalist 137:27-49.

Deacon, J., W. Bradley y K. Larsen. 1964. Ecological distribution of the mammals of Clark Canyon, Charleston Mountains, Nevada. Journal of Mammology 45:397-409.

Escalante, T., D. Espinosa y J. Morrone. 2002. Patrones de distribución geográfica de los mamíferos terrestres de México. Acta Zoológica Mexicana, nueva serie 87:4765.

Fa, E. y L. Morales. 1993. Patterns of Mammalian Diversity in México. In Biological diversity of México: origins and distribution, T. Rammamorthy, R. Bye, A. Lot, and T. Fa (eds.). Oxford University.Oxford. p.319-361.

Fleming, T. 1973. Number of mammal species in North and Central American forest communities. Ecology 54:555-563.

Guerrero, S., J. Téllez y R. Amparan. 1995. Los mamíferos de Jalisco: Análisis Zoogeográfico. Biotam 6: 13-29.

Guerrero, S. y F. Cervantes. 2003. Lista comentada de los 
mamíferos terrestres del Estado de Jalisco, México. Acta Zoológica Mexicana, nueva serie 89:93-110.

Graham, G. 1983. Changes in bat species diversity along an elevational gradient up the Peruvian Andes. Journal of Mammalogy 64:559-571.

Hawkins, B., R. Field, H. Cornell, D. Currie, J. Guégan, D. Kaufman, J. Kerr, G. Mittelbach, T. Oberdorff, E. O’Brien, E. Porter y J. Turner. 2003. Energy, water, and broad-scale geographic patterns of species richness. Ecology 84: 3105-3117.

INEGI. 1981. Síntesis geográfica de Jalisco. Secretaría de Planeación y Presupuesto, México. 306 p.

Illoldi, P., M. Linaje y V. Sánchez-Cordero. 2002. Distribución de los mamíferos terrestres del Golfo de California, México. Anales del Instituto de Biología, Universidad Nacional Autónoma de México, serie zoología 73:213-224.

Iñíguez, L.y E. Santana. 2005. Análisis mastofaunístico del estado de Jalisco. In Contribuciones mastozoológicas en homenaje a Bernardo Villa, V. Sánchez-Cordero y R. Medellín (eds.). Instituto de Biología, UNAM, Instituto de Ecología, UNAM y Comisión Nacional para el Conocimiento y Uso de la Biodiversidad, México, D. F. p. 251- 258.

Kaufman, D. 1995. Diversity of new world mammals: universality of the latitudinal gradients of species and bauplans. Journal of Mammalogy 76:322-334.

Kaufman, D. y M. Willig. 1998. Latitudinal patterns of mammalian species richness in the New World: the effects of sampling method and faunal group. Journal of Biogeography 25:795-805.

Kelt, D., K. Rogovin, G. Shenbrot y J. Brown. 1999. Patterns in the structure of Asian and North American desert small mammal communities. Journal of Biogeography 26:825-841.

Kerr, J. y L. Packer. 1997. Habitat heterogeneity as a determinant of mammal species richness in highenergy regions. Nature 385: 252-254.

Kohlmann, B. y S. Sánchez-Colón. 1984. Estudio aerográfico del género Bursera Jacq. ex L. (Burseraceae) en México: una síntesis de métodos. In Métodos cuantitativos en la biogeografía, E. Escurra, M. Equihua, B. Kohlmann y S. Sánchez-Colón. MAB/ UNESCO e Instituto de Ecología, México, D. F. p. 41-120.

Lobo, J., I. Castro y J. Moreno. 2001. Spatial and environmental determinants of vascular plant species richness distribution in the Iberian Peninsula and Balearic Islands. Biological Journal of The Linnean Society 73:233-253.

Lobo, J. y F. Martín-Piera. 2002. Searching for a predictive model for species richness of Iberian duna beetle base on spatial and environmental variables. Conservation Biology 16: 158-173.

Lomolino, M. 2001. Elevation gradients of species-density: historical and prospective views. Global Ecology \& Biogeography 10: 3-13.

López, A. 2001. Patrones de distribución de la riqueza de especies de la familia Muridae (Mammalia: Rodentia) en el estado de Jalisco. Tesis, Centro Universitario de Ciencias Biológicas y Agropecuarias, Universidad de Guadalajara, Jalisco. 62 p.

López-Wilchis, R., J. López y M. Sánchez. 1998. Specimens of mammals from México in collections in the United States and Canada. Journal of Mammalogy 79:1029-1037.

Matson, J. 1982. Numerical analysis of rodent distributional patterns in Zacatecas, México. Journal of Mammalogy 63:73-84.

Maraña-Peña, M. 1999. Patrones de distribución de la riqueza de especies de carnívoros (Mammalia: Carnivora) en el estado de Jalisco. Tesis, Centro Universitario de Ciencias Biológica y Agropecuarias, Universidad de Guadalajara, Jalisco. 41 p.

McCune, B. 1994. Improving community analysis with the Beals smoothing function. Ecoscience, 1:82-86.

McCune, B. y E. Beals. 1993. History of the development of Bray-Curtis Ordination. In John T. Curtis: Fifty years of Wisconsin Plant Ecology, J. Fralish, R. McIntosh and O. Loucks (eds.). The Wisconsin Academy of Sciences, Arts \& Letters, Madison. p. 67-69.

McCune, B. y J. Grace. 2002. Analysis of ecological communities. MjM Software Design, Gleneden Beach, Oregon. 300 p.

McCune, B. y M. Mefford. 1999. PC-ORD for Windows. Multivariate analysis of ecological data version 4.10. MjM Software Design, Gleneden Beach, Oregon.

Mittelbach, G., C. Steiner, S. Scheiner, K. Gross, H. Reynolds, R. Waide, M. Willig, S. Dodson y L. Gough. 2001. What is the observed relationship between species richness and productivity? Ecology82: 23812396.

Morrone, J. y A. Ruggiero. 2000. Como planificar un análisis biogeográfico. Dugesiana 7:1-8.

Morrone, J. y T. Escalante. 2002. Parsimony analysis of endemicity (PAE) of Mexican terrestrial mammals at different area units: when size matters. Journal of Biogeography 29: 1095-1104.

Murguía, M. y F. Rojas. 2001. Biogeografía cuantitativa. In Introducción a la biogeografía en Latinoamérica: teorías, conceptos, métodos y aplicaciones, B. J. Llorente y J. Morrone (eds.). Las Prensas de Ciencias. Universidad Nacional Autónoma de México, México, D.F. p.39-47. 
O’Hara, T. y G. Poore. 2000. Patterns of distribution for southern Australian marine echinoderms and decapods. Journal of Biogeography 27:1321-1335.

Ortega, J. y H. Arita. 1998. Neotropical-Nearctic limits in middle America as determined by distributions of bats. Journal of Mammalogy 79:772-783.

Owen, J. G. 1990. Patterns of mammalian species richness in relation to temperature, productivity, and variance in elevation. Journal of Mammalogy 71:01-13.

Palomera-García, C., E. Santana y R. Amparan-Salido. 1994. Patrones de distribución de la avifauna en tres estados del occidente de México. Anales del Instituto de Biología, Universidad Nacional Autónoma de México, serie zoología 65:137-175.

Porter, W., S. Buraju, W. Stewar y N. Ramankutty. 2000. Calculating climate effects on birds and mammals: impacts on biodiversity, conservation, populations parameters and global community structure. American Zoologist 40: 597-630.

Prieto, M. y V. Sánchez-Cordero. 1993. Sistemas de información geográfica: un caso de estudio en Veracruz. In Avances en el estudio de los mamíferos de México Publicaciones Especiales, vol. 1, R. Medellín y G. Ceballos (eds.). Asociación Mexicana de Mastozoología, México, D. F. p. 455-464.

Ramírez-Pulido J., J. Arroyo-Cabrales y A. CastroCampillo. 2005. Estado actual y relación nomenclatural de los mamíferos terrestres de México. Acta Zoológica Mexicana, nueva serie 21: 21-82.

Rose, M. y G. Polis. 1998. The distribution and abundance of coyotes: the effects of allochthonous food subsidies from the sea. Ecology79: 998-1007.

Rosenzweig, M. 1992. Species diversity gradients; we know more and less than we thought. Journal of Mammalogy 73: 715-730.

Rzedowski, J. 1986. Vegetación de México. Limusa, México, D.F. 432 p.

Sánchez, O. 1993. Análisis de algunas tendencias ecogeográficas del género Reithrodontomys (Rodentia: Muridae) en México. In Avances en el estudio de los mamíferos de México, Publicaciones Especiales, vol. 1, R. Medellín y G. Ceballos (eds.). Asociación Mexicana de Mastozoología, México, D. F. p. 25-44.

Scott M., F. Davis, B. Csuti, R. Noss, B. Butterfield, C.
Groves, H. Anderson, S. Caicco, F. D'erchia, T. Edwards, J. Ullimany G. Wright. 1993. Gap analysis: a geographic approach to protection of biological diversity. Wildlife Monographs 123:1-41.

Steiner, C. y M. Leibold. 2004. Cyclic assembly trajectories and scale-dependent productivity-diversity relationships. Ecology 85: 107-113.

Stow, A. 1993. The role of geographic information systems for landscape ecological studies. In Landscape ecology and geographic information systems, R. HainesYoung, D. Green, and S. Cousins. Taylor \& Francis, London. p. 11- 21.

Ter Braak, C. y I. Prentice. 1988. A theory of gradient analysis. Advances in Ecological Research 18: 271317.

Villaseñor, J., J. Meave, E. Ortiz y G. Ibarra-Manríquez. 2003. Biogeografía y conservación de los bosques tropicales húmedos de México. In Una perspectiva latinoamericana de la biogeografía, J. Morrone y J. Llorente (eds.). Las Prensas de Ciencias, Universidad Nacional Autónoma de México México, D.F. p. 209216.

Virgós, E., T. Romero y J. Mangas. 2001. Factors determining "gaps" in the distribution of a small carnivore, the common genet (Genetta genetta), in central Spain. Canadian Journal of Zoology 79:15441551.

Whittaker, R., K. Willis y F. Field. 2001. Scale and species richness: towards a general, hierarchical theory of species diversity. Journal of Biogeography 28:453470.

Williams, S., H. Marsh y J. Winter. 2002. Spatial scale, species diversity, and habitat structure: small mammals in Australian tropical rain forest. Ecology 83:13171329.

Willis, K. y R. Whittaker. 2002. Species diversity-scale matters. Science 295: 1245-1248.

Willig, M., D. Kaufman y R. Steven. 2003. Latitudinal gradients of diversity: patterns, process, scale, and synthesis. Annual Review of Ecology and Systematics 34:273- 309.

Zamudio, R. S., F. F. Guevara y J. A. Pérez de la Rosa. 1987. Guía de excursiones botánicas en México. VII. IBUG, Universidad de Guadalajara, Jalisco. 173 p. 